\title{
Generation of the Solar Subsurface Shear
}

\author{
K. L. Chan \\ Mathematics Department, The Hong Kong University of Science 8 \\ Technology, Clear Water Bay, Hong Kong
}

\begin{abstract}
It is well known that a thin layer of angular velocity shear exists just below the solar surface. We propose that this layer is primarily generated by the radial-meridional component of the Reynolds stress. This Reynolds stress component is created by a characteristic upward-equatorward (or equivalently, downward-poleward) correlation of the turbulence velocity over a region in which the shear layer is embedded. Using 2D and 3D numerical experiments, we illustrate that this correlation is caused by vortices that get sucked down from the surface and turn aligned with the rotation vector (a la Taylor columns).
\end{abstract}

\section{Introduction}

Helioseismology results persistently show the existence of a thin shear layer at the top of the solar convection zone, just beneath the solar surface. The feature is most prominent in lower latitudes. An earlier proposal to explain this phenomenon is based on direct angular momentum conservation (Foukal \& Jokipii 1975, Gilman \& Foukal 1979). However, it is obvious that in a rotating convection zone, simple application of angular momentum conservation do not yield the full answer (otherwise, the angular velocity distribution inside the sun would be totally different form what is being observed). Furthermore, there has been no criteria provided to justify the restrained application of simple conservation in a thin subsurface layer. Such an explanation is therefore rather incomplete. It is necessary to investigate the dynamical process in greater detail.

\section{The New Proposal}

Based on observing a large number of numerical experiments on f-plane convection (see Figure 1), we have made the proposal that the subsurface shear layer is generated by the divergence of the radial-meridional component of the Reynolds stress (Chan 2001). This divergence is in balance with the meridional Coriolis force created by the zonal mean flow; the result is the following relationship

$$
\overline{v_{\phi}} \approx \frac{1}{2 \rho \Omega \cos \theta} \cdot \frac{\partial}{\partial r}\left(\overline{\rho v_{r} v_{\theta}}\right)
$$

where $\Omega$ is the angular velocity, $\theta, \phi$, and $r$ are the colatitude (north to south), azimuthal angle (west to east), and the radius, respectively. The sharp negative 

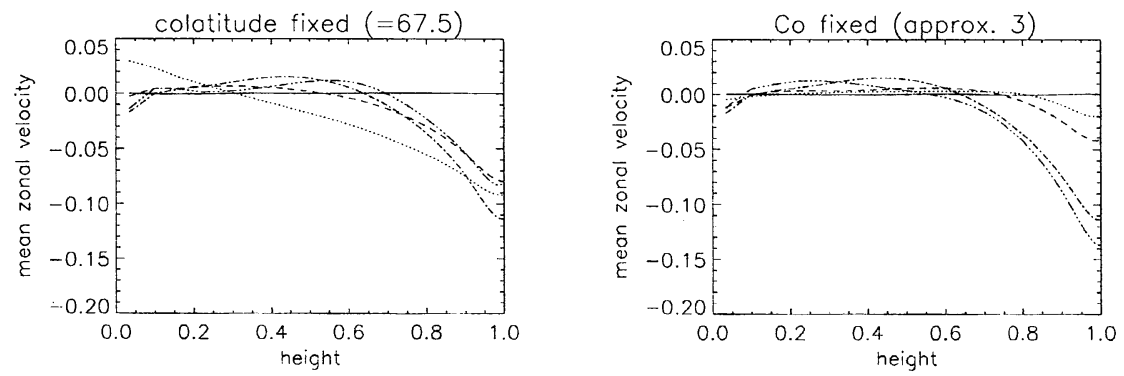

Figure 1. Vertical profiles of the mean zonal velocity. Left panel: Cases at the same colatitude but with different rotation rates. The dotted, dashed, dot-dashed, and triple-dot-dashed curves are for Coriolis numbers $0.8,1.5,2.9$, and 5.8 , respectively. The Coriolis number $C o$ is defined as $\Omega L / V$ where $L$ and $V$ are characteristic length and velocity scales, respectively. Right panel: Cases with same rotation rate but different colatitudes $\theta$. The dotted, dashed, dot-dashed, and triple-dot-dashed curves are for $\theta=22.5,45,67.5,78.75$ degrees, respectively. The key common feature is the development of a dip near the top of the convection zone (when $C o$ is larger than 1).

dip of mean zonal velocity towards the top of the convection zone is interpreted as representing the observed outward drop of angular velocity. The above equation shows that the dip is induced by the drop of the Reynolds stress component from a peak value (positive in northern hemisphere) near the base of the shear layer (see Figure 2). In this paper, we explore the cause of this peak.

\section{The Dynamical Process}

In an earlier paper, Brummell et al. (1996), from observing their numerical simulations, reported the generation of a radial-meridional velocity correlation through the alignment of downflow columns with the rotation vector $\vec{\Omega}$. Such alignment is compatible with the Taylor-Proudman constrain. However, it would only produce a downward-equtorward correlation, in opposite to the kind of correlation we need for generating the surface shear layer. According to our own simulations, the velocity vectors near the surface generally do not align with the rotation axis (see Figure 3). Instead, they tend to be in perpendicular directions. This would create the kind of correlation we get.

There are two ways (or views) to conceptualize this phenomenon. First, one can think of the perpendicular motion as vortices being sucked in by the down flows (initially low-pressure centers with little motion along the rotation axis). Second, one can think of the perpendicular motion as convective rolls in alignment with the rotation axis (this is an alternative way to satisfy the TaylorProudman constrain). In the deeper region, the width scale of the vortices shrinks to very small sizes (due to the stratification and the contraction of the 

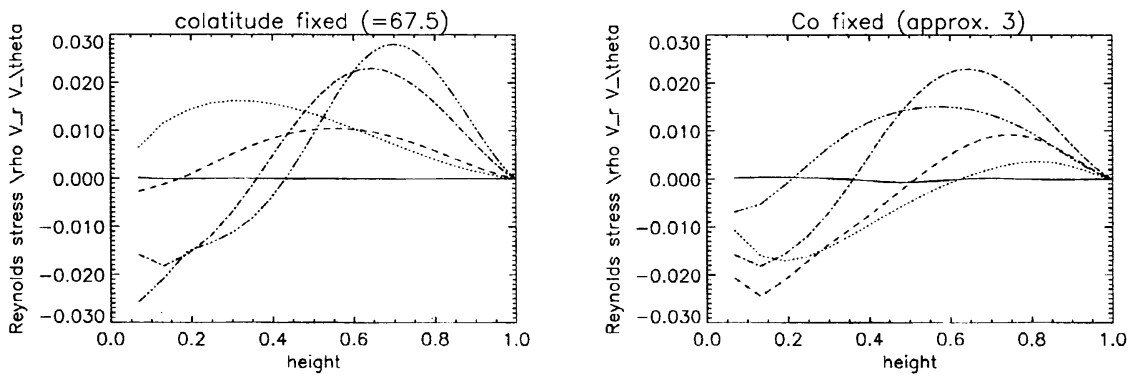

Figure 2. Vertical profiles of the radial-meridional component of the turbulence Reynolds stress for cases corresponding to those described in Figure 1. The key common feature is the positive peak near the top of the convection zone.
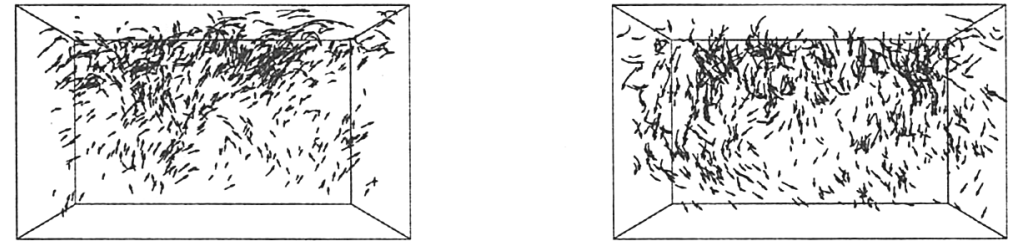

Figure 3. An example of the flow field of a 3D calculation. The horizontal direction is pole-equator oriented. Left panel: Only upward streamlines are plotted. Right panel: Only downward streamlines are plotted. The region is at $\theta=67.5^{\circ}$ and the Coriolis number is close to 3. $\vec{\Omega}$ is tilted upward towards left (in the 2nd quadrant).

Rossby radius $\sim$ r.m.s. $v / \Omega$ ); this quickens the dissipation of the vortical motions and the tilted downflow columns become the dominant feature.

To illustrate the importance of the vortical motion, we performed a 2D numerical experiment which is essentially identical to one of the $3 \mathrm{D}$ experiments except the missing dimension. Vorticity in alignment with $\vec{\Omega}$ cannot exist in the 2D situation, but downflow columns can (see Figure 4). The tilted down flows only generate a downward-equatorward velocity correlation and a positive hump in the mean zonal velocity distribution (see Figure 5).

Acknowledgments. This research is supported by the Research Grant Council of Hong Kong. 

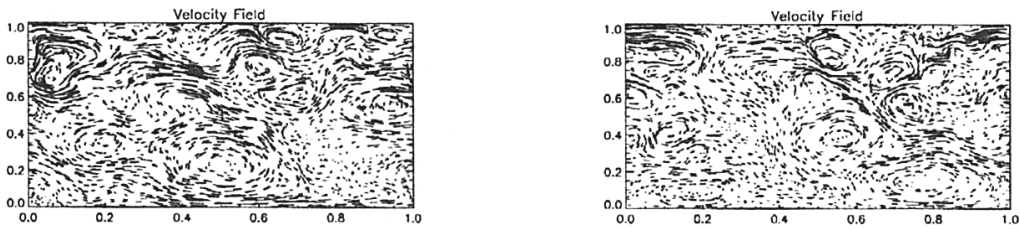

Figure 4. Two examples of streamline distributions in the 2D simulation. The downflows generate a downward-equatorward velocity correlation.
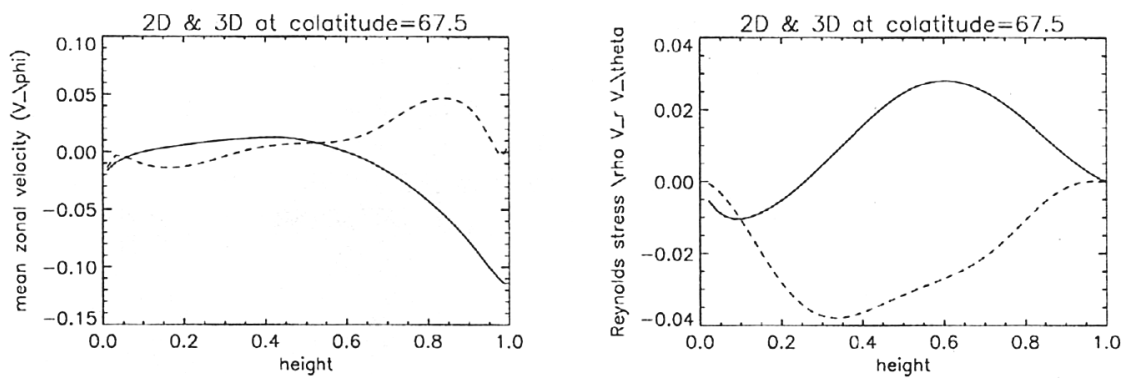

Figure 5. Comparison of 2D (dashed curves) and 3D (solid curves) results. Left panel: Vertical profiles of the mean zonal velocity. Right panel: Vertical profiles of the radial-meridional component of the turbulence Reynolds stress.

\section{References}

Brummell, N. H., Hurlburt, N. E., \& Toomre, J. 1996, ApJ, 473, 494

Chan, K. L. 2001, ApJ, in press

Foukal, P., \& Jokipii, J. R. 1975, ApJ(Letters), 199, L71

Gilman, P. A., \& Foukal, P. 1979, ApJ, 229, 1179 